\title{
Global and local contributions to corneal surface curvature: a simple model
}

\section{WF Harris}

\author{
Department of Optometry, Auckland Park Campus, University of Johannesburg, P O Box 524, \\ Auckland Park, 2006 South Africa
}

<wharris@uj.ac.za>

Received 28 February 2008; revised version accepted 31 March 2008

\begin{abstract}
Variations in corneal surface powers reflect variations in the geometry of the cornea. In particular one can regard corneal surface curvature as a combination of local and global effects. A simple mathematical model of the cornea is presented which makes use of measurements of the curvature of the anterior and posterior surfaces to decompose the curvature into global and local contributions. The model gives insight into the source of variations in keratometric measurements, lids and eye turn, perhaps, for global effects and the tear film, perhaps, for local effects. The model also takes account of the thickness of the cornea. A numerical example is presented.
\end{abstract}

Key words: corneal curvature, local, global, dioptric power matrix, variation

\section{Introduction}

Gillan ${ }^{1}$ has recently examined the front- and backsurface powers of a cornea and their variation. He shows that the front surface varies much more than the back surface. While dioptric power is relevant for the optics of the eye it is less directly relevant for understanding the underlying causes of the variation. Indeed it might even be misleading. The purpose of this paper is to show how consideration of surface curvature instead of surface power has the potential to provide insight into these matters. A very simple model is developed that allows one to separate out what one might call global curvature of the cornea from local effects. The model makes use only of measurements of corneal thickness and anterior and posterior surface curvature.

\section{Surface curvature}

The curvature of a surface is simply related to the dioptric power of the surface. In Gaussian optics the power $F$ and curvature $\kappa$ of a surface are related by $F=\kappa \Delta n$

where $\Delta n$ is the index of refraction after the surface minus the index before it. In linear optics this generalizes to ${ }^{2}$

$\mathbf{F}=\kappa \Delta n$.

Here $\mathbf{F}$ is Fick's ${ }^{3}$ and Long's ${ }^{4}$ dioptric power matrix (symmetric and $2 \times 2$ ). Surface curvature $\kappa$, then, differs from dioptric power in the case of surfa-ces only by the scalar factor $\Delta n$. It, therefore, has the same mathematical character as symmetric dioptric power. In particular it, too, is a symmetric $2 \times 2$ matrix. It follows that all the mathematical and graphical techniques developed for symmetric dioptric power can be used directly for surface curvature as well.

In terms of its entries $\kappa_{i j}$ the surface curvature is

$\kappa=\left(\begin{array}{ll}\kappa_{11} & \kappa_{12} \\ \kappa_{21} & \kappa_{22}\end{array}\right)$.

Just as for the $f_{i j}$, the entries of $\mathbf{F}$, the $\kappa_{i j}$ depend on the reference meridian which we take to be horizontal unless otherwise stated. $\kappa_{11}$ is the curvature in the reference meridian; $\kappa_{21}$ represents the torsion in the reference meridian; and $\kappa_{22}$ is the curvature in the meridian orthogonal to the reference meridian. ${ }^{5,6}$ Following what can be done ${ }^{7}$ for $\mathbf{F}$ the curvature $\kappa$ of Equation (3) can be represented as the sum

$\boldsymbol{\kappa}=\kappa_{\mathrm{I}} \mathbf{I}+\kappa_{\mathrm{J}} \mathbf{J}+\kappa_{\mathrm{K}} \mathbf{K}$

of three components of curvature, $\kappa_{\mathrm{I}} \mathbf{I}$, the spherical component, and $\kappa_{\mathrm{J}} \mathbf{J}$ and $\kappa_{\mathrm{K}} \mathbf{K}$ which we shall refer to as the ortho- and oblique antispherical components respectively. Here

$\mathbf{I}=\left(\begin{array}{ll}1 & 0 \\ 0 & 1\end{array}\right), \mathbf{J}=\left(\begin{array}{cc}1 & 0 \\ 0 & -1\end{array}\right)$ and $\mathbf{K}=\left(\begin{array}{ll}0 & 1 \\ 1 & 0\end{array}\right)$ 
$\kappa_{\mathrm{I}}, \kappa_{\mathrm{J}}$ and $\kappa_{\mathrm{K}}$ are three scalars, the spherical and the ortho- and oblique antispherical coefficients of curvature.

\section{A simple model}

Consider a soap bubble on the bowl of a toy pipe. The curvature of the bubble can be varied globally by blowing more air into, or releasing air from, the bubble through the pipe. The curvatures of the two surfaces (the inside and outside surfaces) are approximately equal and remain so. However one can also imagine local effects which cause differences in the two curvatures and their variation: ripples, for example, confined to one of the surfaces. They would cause differences in measurements of curvature of the two surfaces and its variation. This suggests one might distinguish global and local effects, the former being dependent on the degree to which the two surface curvatures match and the latter the degree to which they differ.

Let one surface have curvature $\boldsymbol{\kappa}_{1}$ and the other curvature $\kappa_{2}$. One can think of two contributions to the curvature of a surface, the global $\kappa_{\mathrm{m}}$ and the local $\kappa_{\mathrm{d}}$ curvatures. In particular, for the second surface,

$\boldsymbol{\kappa}_{2}:=\boldsymbol{\kappa}_{\mathrm{m}}+\boldsymbol{\kappa}_{\mathrm{d} 2}$.

If we define

$\boldsymbol{\kappa}_{\mathrm{d} 2}:=\frac{1}{2}\left(\boldsymbol{\kappa}_{2}-\boldsymbol{\kappa}_{1}\right)$

then we have

$\boldsymbol{\kappa}_{\mathrm{m}}=\frac{1}{2}\left(\boldsymbol{\kappa}_{2}+\boldsymbol{\kappa}_{1}\right)$.

Interchanging subscripts 1 and 2 we obtain corresponding equations for the first surface; it follows that

$\kappa_{\mathrm{d} 1}=-\kappa_{\mathrm{d} 2}$.

We regard $\kappa_{\mathrm{m}}$, defined by Equation (7), as a measure of the coordinated behaviour of the two surfaces of the bubble, the net curvature of the bubble itself or as a whole. On the other hand we can regard either $\kappa_{\mathrm{d} 2}$ or $\kappa_{\mathrm{d} 1}$ as a measure of the degree of independence of the two surfaces, a measure of small-scale effects. $\boldsymbol{\kappa}_{\mathrm{d} 2}$ expresses the small-scale effects as the curvature of the posterior surface relative to the curvature of the anterior surface; $\boldsymbol{\kappa}_{\mathrm{d} 1}$ does the opposite.

In our discussion so far the thickness of the wall of the bubble has played no explicit role. Implicitly we have assumed the thickness to be negligible. For global effects we have taken the curvatures of the surfaces of the bubble to be the same. In the case of the cornea the thickness may not be negligible; it is roughly $0.5 \mathrm{~mm}$ while the radius of curvature is around $7 \mathrm{~mm}$. This suggests that thickness might matter and that it might be appropriate to attempt to account for it in the model. In order to gain clarity on how we might do so we look first at a two-dimensional model of the cornea before generalizing to three dimensions.

\section{A two-dimensional model}

Consider a cornea of thickness $t$ (Figure 1). Because of the thickness the curvatures of the two surfaces $\mathrm{S}_{1}$ and $\mathrm{S}_{2}$ are not directly comparable. In order to make them comparable we shall bring them to a common basis by referring them to the mid-corneal surface $S_{m}$. If the surface $S_{1}$ has radius of curvature $r_{1}$ then its curvature is

$\kappa_{1}:=\frac{1}{r_{1}}$.

We define the compensated radius of curvature of the first surface referred to the mid-corneal surface by $r_{1}-\frac{t}{2}$. Hence the compensated curvature of the front surface referred to the mid-surface is

$\kappa_{1 \mathrm{~m}}=\frac{1}{r_{1}-\frac{t}{2}}$

or

$\kappa_{1 \mathrm{~m}}=\frac{1}{\frac{1}{\kappa_{1}}-\frac{t}{2}}$.

Similarly, the compensated radius of curvature of the back surface referred to the corneal mid-surface is $r_{2}+\frac{t}{2}$ from which we obtain the compensated curvature of the back surface referred to the mid-surface,

$$
\kappa_{2 \mathrm{~m}}=\frac{1}{\frac{1}{\kappa_{2}}+\frac{t}{2}}
$$

We now make use of Equations (6) and (7) but with the compensated surface curvatures $\kappa_{1 \mathrm{~m}}$ (Equation (11)) and $\kappa_{2 \mathrm{~m}}$ (Equation (12)) instead of the actual surface curvatures $\kappa_{1}$ and $\kappa_{2}$ :

$\kappa_{\mathrm{m}}:=\frac{1}{2}\left(\kappa_{2 \mathrm{~m}}+\kappa_{1 \mathrm{~m}}\right)$

and

$\kappa_{\mathrm{d} 2}:=\frac{1}{2}\left(\kappa_{2 \mathrm{~m}}-\kappa_{1 \mathrm{~m}}\right)$. 


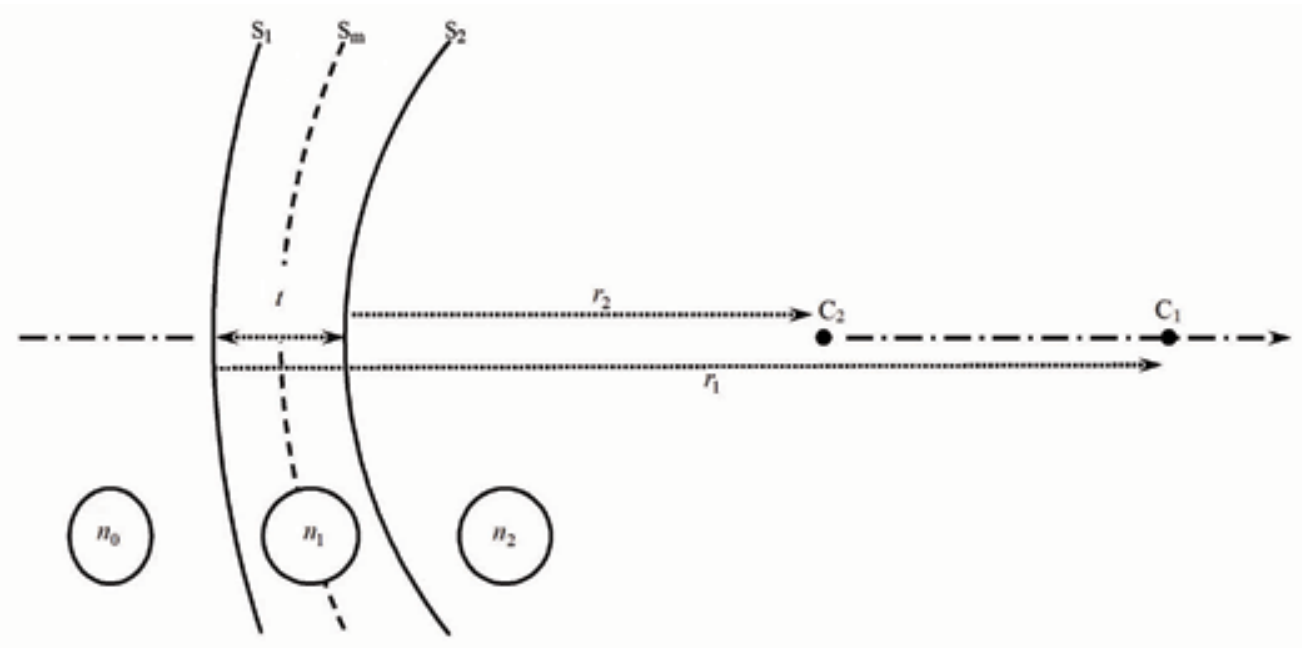

Figure 1 A cornea represented by its anterior and posterior surfaces $\mathrm{S}_{1}$ and $\mathrm{S}_{2} \cdot \mathrm{C}_{1}$ and $\mathrm{C}_{2}$ are the centres of curvature of the anterior and posterior surfaces respectively and $r_{1}$ and $r_{2}$ are the radii of curvature. $t$ is the thickness. $\mathrm{S}_{\mathrm{m}}$ is the mid-corneal surface.

In a sense $\kappa_{\mathrm{m}}$ represents a global curvature of the cornea, a curvature of the cornea as a whole taken at its mid-surface. $\kappa_{\mathrm{d} 2}$ is a measure of how different the curvatures of the two surfaces are. It, too, acts, as it were, at the mid-surface although it is defined in terms of the second surface. It can equally well be defined in terms of its negative (compare Equation (8)), that is, in terms of the first surface.

\section{A three-dimensional model}

The situation in three dimensions is considerably more complicated. However we shall simply generalize the scalar equations for the two-dimensional case to the corresponding matrix equations for three dimensions. The model is simple because we are offering no additional justification. Equations (11) and (12) become

$\boldsymbol{\kappa}_{1 \mathrm{~m}}:=\left(\boldsymbol{\kappa}_{1}^{-1}-\frac{t}{2} \mathbf{I}\right)^{-1}$

and

$\boldsymbol{\kappa}_{2 \mathrm{~m}}:=\left(\boldsymbol{\kappa}_{2}^{-1}+\frac{t}{2} \mathbf{I}\right)^{-1}$

respectively. Reciprocals of scalars have been replaced by inverses of matrices. The identity matrix I is required to make the bracketed term conform for subtraction and addition.

Equations (13) and (14) generalize to

$$
\kappa_{\mathrm{m}}:=\frac{1}{2}\left(\kappa_{2 \mathrm{~m}}+\kappa_{1 \mathrm{~m}}\right)
$$

and

$\boldsymbol{\kappa}_{\mathrm{d} 2}:=\frac{1}{2}\left(\boldsymbol{\kappa}_{2 \mathrm{~m}}-\boldsymbol{\kappa}_{1 \mathrm{~m}}\right)$.

Equation (15) gives the curvature of the first surface of the cornea compensated for thickness; Equation (16) gives the same but for the second surface. Equation (17) defines what we mean by the global curvature of the cornea and Equation (18) defines the local contribution to curvature (of the second surface relative to the first surface), both being referred to the corneal mid-surface. Equation (5) applies except that the left-hand side is $\kappa_{2 \mathrm{~m}}$.

Equations (15) and (16) can be rewritten as

$\boldsymbol{\kappa}_{1 \mathrm{~m}}=\boldsymbol{\kappa}_{1}\left(\mathbf{I}-\frac{t}{2} \boldsymbol{\kappa}_{1}\right)^{-1}$

and

$\boldsymbol{\kappa}_{2 \mathrm{~m}}=\boldsymbol{\kappa}_{2}\left(\mathbf{I}+\frac{t}{2} \boldsymbol{\kappa}_{2}\right)^{-1}$.

Equations (19) and (20) each involve only a single inverse while Equations (15) and (16) each involve two inverses. The latter pair, therefore, has a greater probability of failing in the event that an inverse does not exist. For example, Equations (15) and (16) fail when the surface in question is flat (the curvature is the null matrix) whereas Equations (19) and (20) remain perfectly satisfactory. Equations (15) and (16) also fail when the surface is 
cylindrical. Of course such circumstances are unlikely in the case of the cornea.

A potential numerical disadvantage Equations (19) and (20) have relative to Equations (15) and (16) is that the curvatures $\kappa_{1}$ and $\kappa_{2}$ have each to be input twice.

\section{An approximation}

The binomial expansion can be applied to Equations (19) and (20) to obtain the approximate equations

$\boldsymbol{\kappa}_{1 \mathrm{~m}} \approx \boldsymbol{\kappa}_{1}\left(\mathbf{I}+\frac{t}{2} \boldsymbol{\kappa}_{1}\right)$

and

$\boldsymbol{\kappa}_{2 \mathrm{~m}} \approx \boldsymbol{\kappa}_{2}\left(\mathbf{I}-\frac{t}{2} \boldsymbol{\kappa}_{2}\right)$

These can be written as

$\kappa_{1 \mathrm{~m}} \approx \kappa_{1}+\frac{t}{2} \kappa_{1}^{2}$

and

$\kappa_{2 \mathrm{~m}} \approx \kappa_{2}-\frac{t}{2} \kappa_{2}^{2}$.

Equations (23) and (24) show compensation for thickness as an added term in curvature squared and proportional to thickness.

Substituting into Equations (17) and (18) and rearranging we obtain the approximations

$\boldsymbol{\kappa}_{\mathrm{m}} \approx \frac{1}{2}\left(\boldsymbol{\kappa}_{2}+\boldsymbol{\kappa}_{1}\right)-\frac{t}{4}\left(\boldsymbol{\kappa}_{2}^{2}-\boldsymbol{\kappa}_{1}^{2}\right)$

and

$\kappa_{\mathrm{d} 2} \approx \frac{1}{2}\left(\boldsymbol{\kappa}_{2}-\kappa_{1}\right)-\frac{t}{4}\left(\boldsymbol{\kappa}_{2}^{2}+\boldsymbol{\kappa}_{1}^{2}\right)$.

The approximate expressions contain no inverses and so always work.

\section{Concluding remarks}

A simple model has been devised here to make use of measurements of corneal surface powers and thickness to give what we call the global (Equation (17)) and local (Equation (18)) components of the curvature of a corneal surface. Both components are referred to the mid-surface of the cornea and in a sense are regarded as acting there. We have not attempted to explore these concepts beyond their mathematical definitions. However, we regard the global component as representative of a macroscopic portion (say $2 \mathrm{~mm}$ or more) of the cornea as a whole and the local curvature as a consequence of more microscopic effects perhaps an order of magnitude smaller in scale. One imagines that lids and eye turn might contribute to the former and the tear film or phenomena closer to cellular level might contribute to the latter. The model may, therefore, give insight into the relevance of such effects.

Interestingly the equations for surface curvature compensated for actual thickness $t$ are essentially the same as the equations involved in step-along vergence calculations ${ }^{8,9}$ across a homogeneous gap of reduced thickness $t / 2$. The equations for $\kappa_{1 \mathrm{~m}}$ and $\kappa_{1 \mathrm{~m}}$ (Equations (11), (15) and (19)) correspond to a forward step and those for $\kappa_{2 \mathrm{~m}}$ and $\kappa_{2 \mathrm{~m}}$ (Equations (12), (16) and (20)) correspond to a backward step.

Examination of the dioptric powers of the front and back surfaces, as opposed to the curvatures, may be misleading in the sense of suggesting small values and little variation of the back surface in comparison with the front surface. There is an order of magnitude difference as is illustrated at the end of the numerical example in the Appendix. On the other hand when curvatures are examined instead, particularly compensated for curvature, we see a different picture; magnitudes turn out to be comparable (as in the example in the Appendix).

In many cases (such as that treated in the Appendix) it seems possible to get away with using approximate expressions (Equations (25) and (26)) for the global and local curvatures. A much grosser approximation (Equations (7) and (6)) ignores thickness altogether.

The intention here has been to attempt to extract additional information from relatively simple clinical measurements made on the cornea. The model is extremely simple and, no doubt, can be criticized on many grounds. Certainly any conclusions drawn from it need to be interpreted with due caution.

A paper is in preparation ${ }^{10}$ that applies the method developed here to analyze the cornea of a keratoconic eye.

\section{Acknowledgements}

I thank W D H Gillan for discussions and for commenting on the manuscript.

\section{Appendix: a numerical example}

Consider a cornea of thickness $0.5 \mathrm{~mm}$. The front surface has radii of curvature $7.80 \mathrm{~mm}$ along a principal meridian at $15^{\circ}$ and $7.65 \mathrm{~mm}$ along the other principal meridian. (These figures are taken from an example in the text by Bennett and Rabbetts ${ }^{11}$.) The back surface has radii of curvature $8.20 \mathrm{~mm}$ along a principal meridian that is horizontal and $8.00 \mathrm{~mm}$ along the vertical meridian. 
We obtain the curvatures along the principal meridians: for the first surface we have $128.2 \mathrm{D}$ along $15^{\circ}$ and $130.7 \mathrm{D}$ and for the second surface $122.0 \mathrm{D}$ along $180^{\circ}$ and $125.0 \mathrm{D}$. This represents the eigenstructures of the two surface curvatures. Solving the reverse eigenvalue problem one obtains the curvature matrices themselves. One does so in exactly the same way as one obtains the dioptric power matrix from the principal meridional

powers. ${ }^{12}$ The result is the curvature of the first surface

$$
\kappa_{1}=\left(\begin{array}{cc}
128.37 & -0.63 \\
-0.63 & 130.55
\end{array}\right) \quad D
$$

and the curvature of the second surface

$$
\kappa_{2}=\left(\begin{array}{cc}
121.95 & 0 \\
0 & 125.00
\end{array}\right) \text { D. }
$$

Equations (15) or (19) give the front-surface curvature compensated for thickness,

$\kappa_{1 \mathrm{~m}}=\left(\begin{array}{cc}132.63 & -0.67 \\ -0.67 & 134.96\end{array}\right) \mathrm{D}$.

Compensation increases the magnitudes of the entries in the matrix by some 4\%. Equations (16) or (20) give the back-surface curvature compensated for thickness,

$$
\kappa_{2 \mathrm{~m}}=\left(\begin{array}{cc}
118.34 & 0 \\
0 & 121.21
\end{array}\right) \text { D. }
$$

Compensation decreases the magnitudes by roughly the same proportion. Then the global curvature of the cornea is given by Equation (17),

$$
\kappa_{\mathrm{m}}=\left(\begin{array}{cc}
125.49 & -0.34 \\
-0.34 & 128.08
\end{array}\right) \mathrm{D} \text {, }
$$

and the local curvature by Equation (18),

$$
\kappa_{\mathrm{d} 2}=\left(\begin{array}{cc}
-7.14 & 0.34 \\
0.34 & -6.87
\end{array}\right) \text { D. }
$$

The eigenstructures of these matrices give the curvatures in a form perhaps more familiar to the clinician: the cornea's global curvature is $125.44 \mathrm{D}$ along $7.25^{\circ}$ and $128.13 \mathrm{D}$ and the local curvature for the second surface is $-7.37 \mathrm{D}$ along $146.03^{\circ}$ and $-6.65 \mathrm{D}$. The corresponding global radii of curvature are $7.972 \mathrm{~mm}$ along $7.25^{\circ}$ and $7.805 \mathrm{~mm}$.

From the approximate equations (Equations (23) to (26)) one obtains similar results:

$$
\kappa_{1 \mathrm{~m}}=\left(\begin{array}{cc}
132.49 & -0.67 \\
-0.67 & 134.81
\end{array}\right) \mathrm{D}
$$

$$
\begin{aligned}
& \kappa_{2 m}=\left(\begin{array}{cc}
118.23 & 0 \\
0 & 121.09
\end{array}\right) D \\
& \kappa_{m}=\left(\begin{array}{cc}
125.36 & -0.33 \\
-0.33 & 127.95
\end{array}\right) \quad D \\
& \kappa_{d 2}=\left(\begin{array}{cc}
-7.13 & 0.33 \\
0.33 & -6.86
\end{array}\right) D
\end{aligned}
$$

If one ignores thickness one obtains global curvature

$\kappa_{\mathrm{m}}=\left(\begin{array}{cc}125.16 & -0.31 \\ -0.31 & 127.78\end{array}\right) \mathrm{D}$

via Equation (7) and local curvature

$\kappa_{\mathrm{d} 2}=\left(\begin{array}{cc}-3.21 & 0.31 \\ 0.31 & -2.78\end{array}\right) \mathrm{D}$

\section{via Equation (6).}

For the global curvature the curvature coefficients of the cornea are $\kappa_{\mathrm{I}}=126.66 \mathrm{D}$, the spherical coefficient, $\kappa_{\mathrm{J}}=-1.29 \mathrm{D}$, the ortho-antispherical coefficient, and $\kappa_{\mathrm{K}}=-0.33 \mathrm{D}$, the oblique antispherical coefficient. For the local component of curvature the coefficients are $\kappa_{\mathrm{I}}=-6.99 \mathrm{D}, \kappa_{\mathrm{J}}=-0.14 \mathrm{D}$ and $\kappa_{\mathrm{K}}=0.33 \mathrm{D}$ respectively.

If we take the indices of refraction to be $n_{0}=1$ (air in front of the eye), $n_{1}=1.376$ (the cornea) and $n_{2}=1.336 \quad$ (the aqueous) we obtain the following principal meridional surface powers of the cornea: $48.21 \mathrm{D}$ along $15^{\circ}$ and $49.15 \mathrm{D}$ for the first surface and $-4.88 \mathrm{D}$ and $-5.00 \mathrm{D}$ for the back surface.

\section{References}

1. Gillan WDH. Variation in surface power and thickness of a moderately keratoconic cornea. S Afr Optom 200867 4-10.

2. Harris WF. The second fundamental form of a surface and its relation to the dioptric power matrix, sagitta and lens thickness. Ophthal Physiol Opt 19899 415-419.

3. Blendowske, R. Hans-Heinrich Fick: early contributions to the theory of astigmatic systems. S Afr Optom 200362 105-110.

4. Long WF. A matrix formalism for decentration problems. Am J Optom Physiol Opt 197653 27-33.

5. Harris WF. Elements of the dioptric power matrix and the concept of torsional power: a reinterpretation. Optom Vis Sci 199067 36-37.

6. Harris WF. Signs of surface torsion and torsional dioptric power. Optom Vis Sci 199875 670-673.

7. Harris WF. Power vectors versus power matrices, and mathematical nature of dioptric power. Optom Vis Sci 200784 1060-1063. 
8. Keating MP. Geometric, Physical, and Visual Optics, 2nd ed. Butterworth-Heinemann, Boston, 200289.

9. Harris WF. Step-along vergence procedures in stigmatic and astigmatic systems. Ophthal Physiol Opt 200020487 493.

10. Harris WF, Gillan WDH. Global and local contributions to curvature of a moderately keratoconic cornea. $S$ Afr $\mathrm{Op}$ tom 200867 17-20.

11. Bennett AG, Rabbetts RB. Clinical Visual Optics. Butter worths, London, 1984, 413.

12. Harris WF. Interconverting the matrix and principal meridional representations of dioptric power in general including powers with nonorthogonal and complex principal meridians. Ophthal Physiol Opt 200121 247-252. 